\title{
Perigos do consumo da sibutramina como inibidora de apetite
}

\author{
Dangers of sibutramine consumption as an appetite suppressant \\ Peligros del consumo de sibutramina como supresor del apetito
}

Recebido: 19/09/2021 | Revisado: 30/09/2021 | Aceito: 05/10/2021 | Publicado: 09/10/2021

\author{
Márcio Gomes da Silva \\ ORCID: https://orcid.org/0000-0001-9660-9109 \\ Faculdade Integrada Carajás, Brasil \\ E-mail: marciosaude2010@hotmail.com \\ Thalysson Pereira Rosa \\ ORCID: https://orcid.org/0000-0001-8601-3379 \\ Faculdade Integrada Carajás, Brasil \\ E-mail: thalysonpereirath9@gmail.com \\ Yolanda de Jesus Morais \\ ORCID: https://orcid.org/0000-0001-7105-8267 \\ Faculdade Integrada Carajás, Brasil \\ E-mail: farmaceuticayolandamorais5004@gmail.com
}

\begin{abstract}
Resumo
Introdução: A sibutramina é um medicamento usado para tratar a obesidade, um fármaco de tarja preta, pelo modo como atua no cérebro e atua aumentando rapidamente a sensação de saciedade Consumido na maioria das vezes pelas mulheres na busca pelo corpo perfeito. Objetivo: O objetivo do estudo foi analisar os perigos da sibutramina na perda de peso e os efeitos que ocorrem no organismo. Material e Métodos: O estudo foi conduzido por meio de uma pesquisa bibliográfica e tem como proposta explanar sobre os perigos que ocorrem quando este medicamento é consumido de forma incorreta inibindo o apetite para a perda de peso. Foram selecionados artigos com limite temporal de 2015 a 2021. O estudo ocorreu de fevereiro a julho de 2021, nas bases de dados da Biblioteca Virtual em Saúde (BVS), da Literatura Latino-Americana e do Caribe em Ciências da Saúde (LILACS) e no Scientific Eletronic Library Online (SciELO). Resultados e discussão: A sibutramina é um remédio aprovado e muito consumido pelas pessoas, onde se sentem saciados e perdem peso muito rápido, devido a diminuição do apetite e a queima de gordura. O fármaco tem eficácia comprovada no emagrecimento e o consumo inadequado, com a falta de uma alimentação saudável e atividade física apresentam perigos a saúde do ser humano com vários efeitos adversos. Conclusão: O percentual de pessoas que consomem a sibutramina cresceu nos últimas anos, tudo isso pelo emagrecimento rápido causados pelo sobrepeso e obesidade. $\mathrm{O}$ uso incorreto provoca muitos efeitos adversos graves. Portanto, antes de se automedicar, o paciente deve procurar um médico para começar o tratamento na perda de peso.
\end{abstract}

Palavras-chave: Consumo; Perigos; Inibidor; Sibutramina.

\begin{abstract}
Introduction: Sibutramine is a drug used to treat obesity, a drug with a black stripe, because of the way it acts on the brain and acts quickly increasing the feeling of satiety Consumed most often by women in search of the perfect body. Objective: The aim of the study was to analyze the dangers of sibutramine in weight loss and the effects that occur in the body. Material and Methods: The study was conducted through a literature review and aims to explain the dangers that occur when this drug is consumed incorrectly, inhibiting the appetite for weight loss. Articles with a time limit from 2015 to 2021 were selected. The study took place from February to July 2021, in the databases of the Virtual Health Library (VHL), the Latin American and Caribbean Literature on Health Sciences (LILACS) and in Scientific Electronic Library Online (SciELO). Results and discussion: Sibutramine is an approved medicine and is widely consumed by people, where they feel satiated and lose weight very fast, due to decreased appetite and fat burning. The drug has proven efficacy in weight loss and inadequate consumption, with the lack of healthy eating and physical activity, they present dangers to human health with several adverse effects. Conclusion: The percentage of people who consume sibutramine has grown in recent years, all due to rapid weight loss caused by overweight and obesity. Misuse causes many serious adverse effects. Therefore, before self-medicating, the patient must see a doctor to start the weight loss treatment.
\end{abstract}

Keywords: Consumption; Hazards; Inhibitor; Sibutramine.

\section{Resumen}

Introducción: La sibutramina es un fármaco que se utiliza para tratar la obesidad, un fármaco con una raya negra, por la forma en que actúa sobre el cerebro y actúa rápidamente aumentando la sensación de saciedad. Consumido con mayor frecuencia por mujeres en busca del cuerpo perfecto. Objetivo: El objetivo del estudio fue analizar los peligros 
de la sibutramina en la pérdida de peso y los efectos que se producen en el organismo. Material y métodos: El estudio se realizó a través de una revisión de la literatura y tiene como objetivo explicar los peligros que se producen cuando este fármaco se consume de forma incorrecta, inhibiendo el apetito por adelgazar. Se seleccionaron artículos con plazo de 2015 a 2021. El estudio se llevó a cabo de febrero a julio de 2021, en las bases de datos de la Biblioteca Virtual en Salud (BVS), la Literatura Latinoamericana y del Caribe en Ciencias de la Salud (LILACS) y en Electrónica Científica. Biblioteca en línea (SciELO). Resultados y discusión: La sibutramina es un medicamento aprobado y es ampliamente consumido por personas, donde se sienten saciadas y pierden peso muy rápido, debido a la disminución del apetito y la quema de grasa. El fármaco ha demostrado su eficacia en la pérdida de peso y su consumo inadecuado, siendo la falta de una alimentación saludable y de actividad física peligros para la salud humana con varios efectos adversos. Conclusión: El porcentaje de personas que consumen sibutramina ha crecido en los últimos años, todo debido a la rápida pérdida de peso provocada por el sobrepeso y la obesidad. El mal uso causa muchos efectos adversos graves. Por tanto, antes de automedicarse, el paciente debe acudir al médico para iniciar el tratamiento de adelgazamiento.

Palabras clave: Consumo; Riesgos; Inhibidor; Sibutramina.

\section{Introdução}

A sibutramina é um medicamento usado para tratar a obesidade, pois aumenta rapidamente a sensação de saciedade, evitando que sejam ingeridos alimentos em excesso e, assim, facilita na perda de peso. Além disso, este remédio também aumenta a termogênese, o que também contribui para emagrecimento (Moreira \& Alves, 2015).

A sibutramina é usada na forma de cápsulas e pode ser comprado nas farmácias convencionais em forma de genérico ou com o nome comercial de Reductil, Biomag, Nolipo, Plenty ou Sibus, por exemplo, mediante apresentação de receita médica (Tavares; Angelo \& Souza, 2017).

A sibutramina está indicada para o tratamento de pessoas com obesidade em casos de IMC superior a $30 \mathrm{mg} / \mathrm{m}^{2}$, que estão fazendo acompanhamento com um nutricionista ou um endocrinologista, por exemplo. Cabe pontuar que esse é um fármaco de tarja preta, pelo modo como atua no cérebro. O aviso na embalagem sinaliza que o princípio ativo pode causar problemas à saúde e dependência, especialmente se utilizado sem acompanhamento e inadvertidamente (Magalhães; Dinelly \& Oliveira, 2016).

A sibutramina é um inibidor da recaptação da serotonina e da noradrenalina, com atividade antidepressiva fraca, mas pelo efeito colateral de causar saciedade, é usada como adjuvante no tratamento da obesidade, sempre em combinação com a dieta e a atividade física. Foram descritos efeitos benéficos, como melhora da resistência à insulina, do metabolismo da glicose e da dislipidemia, que podem estar relacionados mais à perda de peso do que à ação intrínseca da droga (James et al., 2017).

Diante dos problemas causados pela obesidade e da busca pelo corpo e estética ideal, muitas pessoas procuram alternativas simples e rápidas para emagrecer. Com isso, os fármacos anorexígenos são vistos como o melhor aliado para combater o excesso de peso, entretanto, seu consumo abusivo e irracional pode gerar riscos à saúde (Moreira \& Alves, 2015), nesse sentido, o objetivo desse artigo é analisar os perigos da sibutramina na perda de peso e os efeitos que ocorrem no organismo.

\subsection{Sibutramina}

A sibutramina é um fármaco utilizado no tratamento da obesidade, com mecanismo de ação diferente da dfenfluramina e d-anfetamina (Heal et al., 1998). Foi desenvolvido inicialmente como antidepressivo no final de 1980. Nos ensaios clínicos foi verificado que o medicamento reduzia o apetite. Então, sob o nome Meridia®, foi comercializado nos EUA e Alemanha.

Cassin (2018) descreve a sibutramina com ação diminuidora de apetite e proporcionando uma sensação de saciedade, foi também utilizada como antidepressivo agindo no sistema nervoso central (SNC) e sobre os neurotransmissores serotonina e 
noradrenalina. O uso dessa medicação iniciou-se em 1997, após a liberação oficial pelo Food and Drug Administration (FDA), nos Estados Unidos (Oliveira et al., 2016).

Consta no Conselho Federal de Farmácia o cloridrato de sibutramina, "um medicamento emagrecedor com registro válido mais antigo em março de 1998". Sua referência é o Reductil (CFF, 2017). Esse medicamento é encontrado no mercado brasileiro de referência, manipulado, similar e genérico. Sua característica é de um pó branco leitoso, com pH 5,2 e solubilidade 2,9mg/ml (Reductil, 2019).

Lima (2017) enfatiza que no Brasil a sibutramina é disponibilizada pelo Sistema único de saúde (SUS), gratuitamente pelo governo para o tratamento de pacientes com obesidade, pois é capaz de ativar na perda de massa corporal, estando ligado a exercícios físicos e alimentação saudável.

A quantidade máxima por receita deve ser de 60 dias de tratamento. No caso da sibutramina, a RDC 133/2016 consolida a determinação da Anvisa em relação à quantidade de medicamento que poderá constar em cada notificação de receita apresentada pelo paciente. A quantidade de medicamento máxima por receituário deve ser igual a 60 (sessenta) dias de tratamento (Brasil, 2016).

No início do tratamento a dose recomendada é de 1 cápsula de $10 \mathrm{mg} /$ dia no período da manhã e no decorrer do tratamento podendo aumentar para $15 \mathrm{mg} / \mathrm{dia}$. A duração do tratamento não pode passar de 2 anos e não é recomendado ao paciente usar mais de 15mg/dia (Andrade et al., 2019).

\subsection{Mecanismo de ação}

Segundo Oliveira et al. (2016), a sibutramina é um dos medicamentos da classe dos inibidores da recaptação de noradrenalina e serotonina (5-HT), com a função de bloquear os receptores pré-sinápticos de noradrenalina e serotonina nos centros de alimentação e saciedade do hipotálamo, avançando os efeitos anorexígenos dos neurotransmissores e provocando a redução de fome. Com isso, asibutramina estimula à saciedade mais rápido e não controla o apetite. Com isso, as pessoas que usam esses medicamentos se alimentam em menor quantidade e se sentem saciados mais rápidos (Dutra; Souza \& Peixoto, 2015). No decorrer do tratamento, o indivíduo se alimenta pouco por se sentir satisfeito, onde o cérebro transmite a mensagem de estômago cheio (Oliveira et al., 2016).

Radaelli, Pedroso \& Medeiros (2016) relata que tem outro efeito que a sibutramina proporciona: aceleração do metabolismo basal, estímulos da termogênese na contribuição da perda de peso e aumento de 100 kcal na energia gasta.

Segundo Marini (2015), a redução do apetite e a saciedade que esses medicamentos provocam, é consequência da substância que ocupa o espaço dos receptores de noradrenalina e serotonina, impedindo que os neurotransmissores entrem nos neurônios.

\subsection{Medicamento mais usado pelos gêneros}

Em uma pesquisa feita por acadêmicos de estéticas em Copacabana (RJ) pelo perfil ideal de corpo, constatou-se que as mulheres utilizavam mais a sibutramina e a faixa etária que prevaleceu foi de 17 a 25 anos. Por mais que tem acesso das informações sobre o risco da automedicação, mesmo assim fazem uso sem orientação médica (Silva et al., 2018).

Costa et al. (2018), apresentou uma pesquisa no município de Bacabal-MA, onde constatou que $84 \%$ dos pacientes eram mulheres de 18 a 35 anos. Tudo isso pela pressão da sociedade pelo corpo perfeito e disputas entre as mulheres. Para ter um resultado mais rápido e fácil, elas escolhem a medicação, sem ao menos praticar atividade física e/ou se alimentarem de forma saudável. Andrade et al. (2019) apontam que não há gênero e nem idade para desenvolverem a obesidade, mas que as mulheres possuem um metabolismo menor que os homens. 


\subsection{Restrições}

Pacientes com depressão, arritmia cardíaca, acidente vascular cerebral e insuficiência cardíaca congestiva não podem fazer uso de sibutramina (Ache, 2019).

Também há casos de restrição para mulheres suspeitas de gravidez, lactantes e de transtornos alimentares. Os pacientes que tiver fazendo uso desse medicamento, não poderá dirigir e nem manusear máquinas, pois suas habilidades e atenção estarão prejudicadas (Reductil, 2019).

Na RDC 52/2016 enfatiza a obrigatoriedade do "Termo de Responsabilidade do Prescritor", onde deve ser preenchido em três vias, ficando uma via arquivada no prontuário do paciente, outra na drogaria e a última com o paciente (Santos \& Belo, 2016).

Neste estudo consta os perigos do consumo desse medicamento e a relevância de fazer uma alimentação equilibrada e praticar exercícios físicos diariamente (Oliveira et al., 2016).

No Brasil, o comércio de medicamentos psicotrópicos é regulado pela Portaria ${ }^{\circ}$ 344/1998 da ANVISA, que estabelece que os medicamentos sujeitos a controle especial, contendo, entre outros, fármacos anorexígenos pertencem à Lista B2, devem ser dispensados mediante apresentação de notificação de receita B (Gonzaga, 2015).

\subsection{Perigos ao uso da sibutramina}

O mal do ser humano é exagerar no consumo desses inibidores de apetite em decorrência da rápida perda de peso e pressa em querer o resultado mais rápido pelo corpo perfeito e não se importar com os males à saúde (OLIVEIRA et al., 2016). O mal consumo desse fármaco pode acarretar hemorragia cerebral e aumento da resistência bacteriana pelo consumo inadequado (Dutra; Souza \& Peixoto, 2015).

Após o consumo da sibutramina apareceram várias reações adversas em $50 \%$ dos indivíduos, as quais $40 \%$ mais detectadas foram: taquicardia, boca seca, insônia, irritabilidade, cefaleia e mal-estar (Santos \& Belo, 2016).

Muitos usuários não chegam a se consultar com um médico e vão direto para a automedicação. Tem drogarias que vendem esse medicamento sem receita levando o usuário a consumir de forma errada (Cassin, 2018).

A importância de se manter uma alimentação equilibrada com um profissional nutricionista e atividades físicas ajudará o paciente a perder mais peso e quando o tratamento for finalizado, continuará controlando o peso com mais facilidade. Se no período do tratamento com o profissional o paciente não seguir esses hábitos, quando for finalizado o consumo, poderá voltar a engordar e até ultrapassar o peso anterior.

\section{Metodologia}

O presente estudo trata-se de uma revisão integrativa sendo fundamentada na pesquisa bibliográfica, que agrupa a produção científica relevante acerca de um tema preestabelecido, ofertando acesso rápido e sintetizado aos resultados científicos de maior acuidade para a área estudada (Broome, 2000) e estabelecida por meio de artigos publicados sobre os perigos do consumo da sibutramina como inibidor de apetite. E para consolidação dela, seguiu-se as seguintes etapas: escolha do tema, levantamento bibliográfico preliminar, formulação do problema, busca de fontes, leitura do material, organização lógica do assunto e por fim redação do texto.

A coleta foi realizada nos períodos de fevereiro a julho de 2021, onde foram investigados estudos publicados nas bases de dados da Biblioteca Virtual em Saúde (BVS), da Literatura Latino-Americana e do Caribe em Ciências da Saúde (LILACS) e no Scientific Eletronic Library Online (SciELO). A pesquisa foi restrita nas línguas portuguesa e inglesa nos períodos de 2015 a 2021. 
Após a fase de pesquisa, os artigos foram salvos no computador, depois foram lidos e em seguida selecionados de acordo com o tema em questão. Por meio dos métodos determinados na pesquisa foram encontrados 40 artigos nas bases de dados citados e excluídos 30 artigos pelos seguintes motivos: estudos duplicados, assuntos não condizentes com o abordado e fora do período temporal citado, onde 10 foram pré-selecionados por meio de títulos e resumos, acondicionados e preparados após ser lidos. Foram escolhidos 6 artigos para a elaboração do referencial teórico.

A Figura 1, apresenta a seleção dos artigos utilizados na revisão.

Figura 1 - Diagrama de fluxo de revisão - integrativa de análise de estudos.

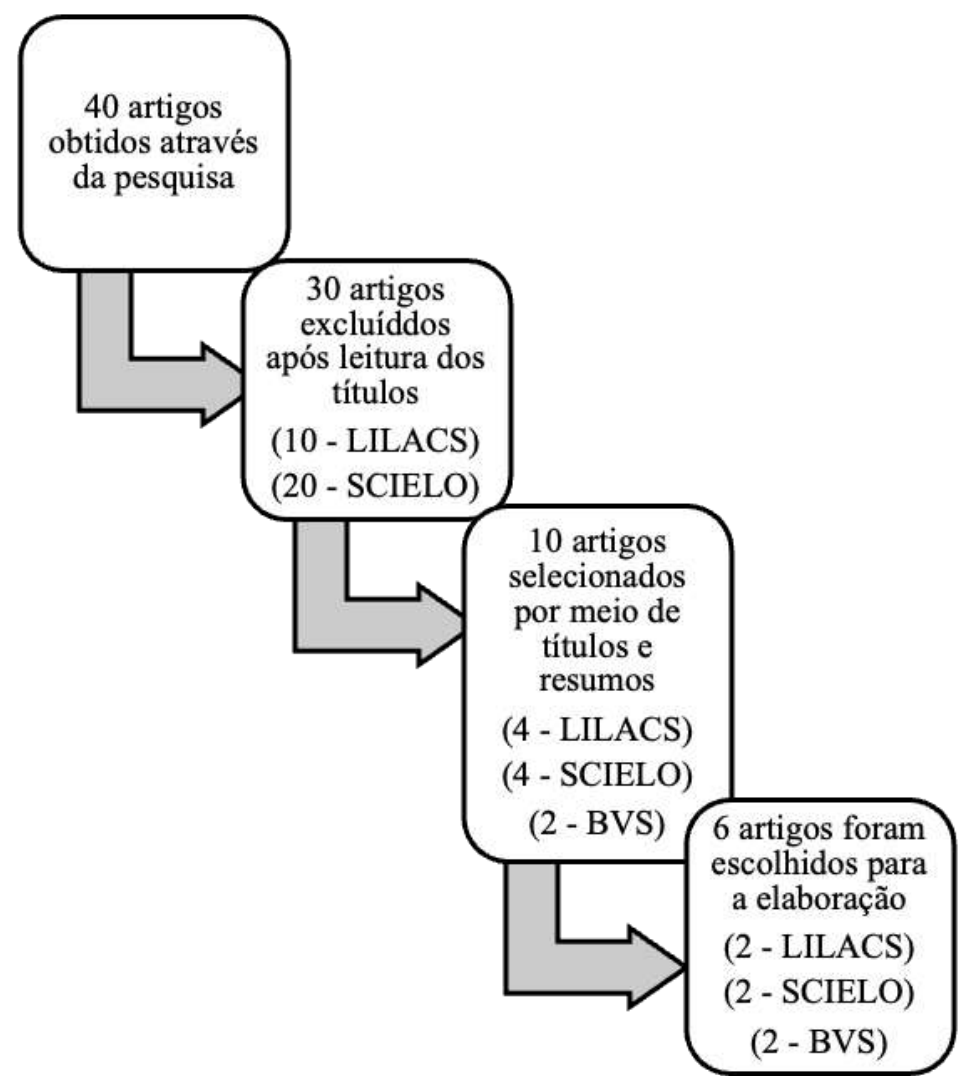

Fonte: Perigos do consumo da sibutramina como inibidora de apetite, Redenção - PA (2021).

\section{Resultados}

A Tabela 1 apresenta detalhes dos 06 artigos selecionados, os estudos selecionados foram publicados entre 2015 e 2019.

Tabela 1- Principais estudos sobre a sibutramina e perigos ao consumo.

\begin{tabular}{|c|c|c|c|}
\hline AUTORES/ANO & OBJETIVO & DESENHO DO ESTUDO & CONSIDERAÇÕES FINAIS \\
\hline Cassin (2018) & $\begin{array}{l}\text { Realizar um estudo descritivo } \\
\text { sobre o perfil de segurança da } \\
\text { sibutramina, bem como } \\
\text { apresentar alternativas } \\
\text { terapêuticas disponíveis para o } \\
\text { tratamento da obesidade no } \\
\text { mercado internacional. }\end{array}$ & $\begin{array}{c}\text { Estudo retrospectivo e } \\
\text { descritivo }\end{array}$ & $\begin{array}{l}\text { O tratamento farmacológico da obesidade } \\
\text { é bastante complexo e possui grandes } \\
\text { desafios a serem superados (a eficácia a } \\
\text { longo prazo e a segurança). Muitos } \\
\text { medicamentos agem por meio de } \\
\text { diferentes mecanismos para controle da } \\
\text { saciedade e da compulsão alimentar, mas } \\
\text { ainda não existe um alvo terapêutico } \\
\text { considerado ideal. }\end{array}$ \\
\hline
\end{tabular}




\begin{tabular}{|c|c|c|c|}
\hline $\begin{array}{l}\text { Dutra, Souza e Peixoto } \\
\text { (2015) }\end{array}$ & $\begin{array}{l}\text { Descrever os riscos do uso } \\
\text { indiscriminado da sibutramina } \\
\text { como inibidor de apetite. }\end{array}$ & Revisão de Literatura & $\begin{array}{l}\text { A sibutramina deve ser utilizada apenas } \\
\text { quando o tratamento não-farmacológico } \\
\text { não funcionar, e em situações especiais } \\
\text { de acordo com o julgamento médico. É } \\
\text { importante que o paciente busque, } \\
\text { primeiramente, redução de peso através } \\
\text { de métodos convencionais, como } \\
\text { reeducação alimentar e prática de } \\
\text { exercícios físicos. }\end{array}$ \\
\hline Andrade et al. (2019) & $\begin{array}{l}\text { Investigar as saídas dos principais } \\
\text { emagrecedores dispensados, } \\
\text { abordando os riscos que essa } \\
\text { medicação pode causar. }\end{array}$ & $\begin{array}{l}\text { Estudo retrospectivo e } \\
\text { transversal }\end{array}$ & $\begin{array}{l}\text { Houve um aumento no controle quanto ao } \\
\text { uso racional e seguro dos emagrecedores } \\
\text { acarretado pela presença do profissional } \\
\text { farmacêutico na drogaria destacando } \\
\text { assim a importância do papel do } \\
\text { profissional e a assistência prestada } \\
\text { quanto aos riscos que essa classe } \\
\text { medicamentosa pode causar a saúde física } \\
\text { e mental quando aderindo de forma } \\
\text { irracional. }\end{array}$ \\
\hline Oliveira et al. (2016) & $\begin{array}{l}\text { Levantar, através da bibliografia, } \\
\text { os efeitos, riscos e benefícios do } \\
\text { uso da sibutramina para o } \\
\text { emagrecimento, resgatando } \\
\text { conceitos já definidos na } \\
\text { literatura. }\end{array}$ & $\begin{array}{l}\text { Revisão bibliográfica } \\
\text { integrativa }\end{array}$ & $\begin{array}{l}\text { A literatura apontou que a substância traz } \\
\text { um perfil de segurança favorável para um } \\
\text { grupo de pacientes obesos que não } \\
\text { apresentam doenças cardiovasculares de } \\
\text { modo que possui benefícios, mas também } \\
\text { apresenta riscos, portanto, seu uso deve } \\
\text { ser fundamentado em critérios adequados. }\end{array}$ \\
\hline Santos e Belo (2016) & $\begin{array}{l}\text { Identificar os principais impactos } \\
\text { no sistema cardiovascular do uso } \\
\text { de inibidores de apetite. }\end{array}$ & Levantamento bibliográfico & $\begin{array}{l}\text { Devido ao crescente número de } \\
\text { indivíduos com sobrepeso e obesidade, } \\
\text { bem como a busca por um } \\
\text { emagrecimento rápido por questões de } \\
\text { saúde e/ou finalidades estéticas, há um } \\
\text { uso descontrolado e irracional dos } \\
\text { inibidores de apetite como anfepramona, } \\
\text { femproporex e mazindol, assim como do } \\
\text { sacietógeno sibutramina. }\end{array}$ \\
\hline Costa et al. (2018) & $\begin{array}{ll}\text { Mostrar os evidentes conflitos de } \\
\text { interesses envolvidos na } \\
\text { discussão e a manipulação de } \\
\text { opinião pelos defensores da } \\
\text { manutenção dos medicamentos. }\end{array}$ & Pesquisa exploratória & $\begin{array}{c}\text { No início de outubro, quando este } \\
\text { fascículo da Brasília Médica estava } \\
\text { fechando, veio a decisão da Anvisa (RDC } \\
52 / 2011 \text {, publicado no Diário Oficial da } \\
\text { União no dia } 10 \text { de outubro de 2011) de } \\
\text { suspender a comercialização dos } \\
\text { medicamentos anoréticos do tipo } \\
\text { anfetamina. }\end{array}$ \\
\hline
\end{tabular}

Fonte: Perigos do consumo da sibutramina como inibidora de apetite, Redenção - PA (2021).

\section{Discussão}

O Instituto Brasileiro de Geografia e Estatística (IBGE) divulgou no final do ano passado, os dados da última Pesquisa Nacional de Saúde, entre 2003 e 2019 a ocorrência de obesidade entre brasileiros acima de 20 anos passou de 12,2 para 26,8\%. O excesso de peso saltou de 43,3 para $61,7 \%$ decorrentes dos estresses e preocupações que a população vem tendo (IBGE, 2020). 
No Brasil, Cassin (2018) descreve um remédio aprovado e muito consumido pelas mulheres brasileiras a “sibutramina” que age no SNC, reduzindo, assim, a fome e onde o impacto no emagrecimento é mais considerável, sobretudo ela é ligada a alguns efeitos adversos. Por isso não deve ser indicada a idosos e indivíduos com diabetes.

Em um estudo bibliográfico e exploratório feito por Dutra, Souza \& Peixoto (2015), os autores puderam constatar que pessoas que usam esses medicamentos se sentem saciados e perdem peso muito rápido, devido a diminuição do apetite e a queima de gordura. Mas quando param de consumir esse fármaco sem a ajuda de um profissional, engordam o dobro de quilos que tinha anteriormente.

A maioria das mulheres querem perder peso sem esforços e utilizam a sibutramina sem ao menos terem conhecimento sobre os perigos do consumo incorreto, sem orientação e acompanhamento. De acordo com Andrade et al. (2019) o consumo inadequado desse medicamento antiobesidade é visto hoje como uma ferramenta indispensável na assistência farmacêutica e para isso o estabelecimento deve vender só com prescrição, com as doses adequadas e duração correta do tratamento, para que a dispensação seja adequada e com a orientação do farmacêutico.

Sendo primordial o conhecimento sobre o consumo de sibutramina, Oliveira et al. (2016) vem com um estudo sobre os perigos de se consumir inadequadamente e a diferença de fazer uma alimentação equilibrada e praticar atividades físicas regularmente durante e depois do tratamento.

Santos \& Belo (2016) tendo como objetivo de sua pesquisa a mesma visão temática que é analisar os perigos da sibutramina na perda de peso e os efeitos que ocorrem no organismo, ambos afirmaram que apareceram várias reações adversas nos indivíduos como: boca seca, taquicardia, mal-estar, insônia e queda de pressão arterial.

A ANVISA reavaliou na sua constatação que os benefícios de consumo a sibutramina eram maiores que os perigos, na condição de ser usada com um profissional responsável e para determinados tipos de pacientes. Só pode ser dispensada na apresentação de Notificação "B2” com o máximo de sessenta dias de tratamento, na dose máxima de 15 mg/dia (ANVISA, 2017).

A obesidade e o conceito de vida fitness, atualmente são assuntos com grande destaque nas mídias sociais. Costa et al. (2018), em seu estudo, observaram que as pessoas estão mais antenadas às redes sociais em relação ao corpo perfeito e a venda de produtos de emagrecimento e sem ter noção da gravidade de se consumir sem a orientação de um profissional especializado na área., os dados encontrados mostraram um aumento do consumo da sibutramina, em decorrência de ter drogarias que ainda vendem sem prescrição e a falta de conscientização do indivíduo em relação aos efeitos adversos que o fármaco pode causar.

\section{Considerações Finais}

Com o crescimento do sobrepeso e obesidade na população brasileira e assim, a busca pelo emagrecimento rápido com finalidades estéticas, aumentou muito o consumo desequilibrado desses inibidores e principalmente da sibutramina. O propósito desse fármaco é na perda de peso, mas causa efeitos adversos graves como mudança no humor, pressão alta, insônia e dependência química e psíquica.

Para afirmar de fato, se a sibutramina é prejudicial à saúde e proibí-la, deveria existir embasamentos maiores de informações científicas sobre o assunto. Independente do parecer final, a intensificação na fiscalização deste fármaco seria uma boa opção para minimizar as irregularidades de alguns prescritores, pacientes e dispensadores. A mobilização do mesmo, através de campanhas, divulgações na mídia, debates e propagandas é indispensável para alertar sobre ética, responsabilidade e uso indiscriminado, como por exemplo, associações medicamentosas com anorexígenos. 
O risco do consumo indiscriminado da sibutramina provocada no organismo é muito grande, já que a administração do medicamento em pessoas com problemas cardíacos pode levar ao derrame e enfarte, além de uma infinidade de efeitos colaterais, levando até a óbito ao paciente.

\section{Implicações práticas}

Recomenda-se a necessidade de estudos complementares quanto a descrição dos reais riscos do uso indiscriminado da sibutramina.

\section{Contribuições dos autores}

MGS e TPR, concebeu a estratégia de pesquisa em conjunto com YJM. MGS e TPR, fez as buscas bibliográficas nas bases de dados, a seleção dos artigos e a extração de dados sob a supervisão de YJM. Os autores leram e aprovaram a versão final do manuscrito. O conteúdo da revisão é de exclusiva responsabilidade individuais dos autores.

\section{Declaração de conflito de interesses}

Os autores declaram que a pesquisa foi conduzida na ausência de quaisquer relações comerciais ou financeiras que possam ser interpretados como um potencial conflito de interesse

\section{Referências}

Alves, M., Teixeira, A. L., de Barros Anastácio, L., da Silva Alves, G. C., Baldoni, N. R., \& Chequer, F. M. D. (2018). Análise dos efeitos adversos associados ao uso da anorexígena sibutramina: revisão sistemática. Journal of Health \& Biological Sciences, 6(3), 313-326. Doi:10.12662/2317-3076jhbs.v6i31588.p313326.2018 .

Andrade, t. b., \& Silva, j. n. d. (2019). Os riscos do uso indiscriminado da sibutramina como inibidor de apetite.

Brasil. Agência nacional de Vigilância Sanitária. (2017). Sibutramina e remédios para emagrecer: entenda. http://antigo.anvisa.gov.br/resultado-debusca?p_p_id=101\&p_p_lifecycle=0\&p_p_state=maximized\&p_p_mode=view\&p_p_col_id=column-

1\&p_p_col_count=1\&_101_struts_action=\%2Fasset_publisher\%2Fview_content\&_101_assetEntryId=3423550\&_101_type=content\&_101_groupId=219201

\&_101_urlTitle=sibutramina-e-remedios-para-emagrecer-entenda\&inheritRedirect=true.

Brasil. Agência nacional de Vigilância Sanitária. RDC reúne regras para venda dos anorexígenos. 2016. http://antigo.anvisa.gov.br/pt BR/noticias//asset_publisher/FXrpx9qY7FbU/content/rdcreuneregrasparavendadosanorexigenos/219201/pop_up?_101_INSTANCE_FXrpx9qY7FbU_viewMode=print\&_ 101_INSTANCE_FXrpx9qY7FbU_languageId=pt_BR.

Broome ME. Integrative literature reviews for the development of concepts. In: Rodgers BL, Knafl KA, editors. Concept development in nursing: foundations, techniques and applications. Philadelphia (USA): W.B Saunders Company; 2000. 231-50 p.

Camelo, A. E. M., Dinelly, C. M. N., \& Oliveira, M. A. S. (2016). Psicotrópicos: perfil de prescrições de benzodiazepínicos, antidepressivos e anorexígenos a partir de uma revisão sistemática. Revista Eletrônica de Farmácia, 13(3), 111-122. https://revistas.ufg.br/REF/article/view/35226/pdf.

Campos, L. S., DE Oliveira, L. A., Da Silva, P. K. P., \& De Paiva, A. M. R. (2014). Estudo dos efeitos da sibutramina. Revista Uningá Review, 20(3). https://www.mastereditora.com.br/periodico/20141130_221647.pdf.

Casulari, L. A., \& Motta, L. (2011). Reflexões sobre os conflitos de interesses entre médicos e a indústria farmacêutica: a propósito da sibutramina. Brasília méd. https://cdn.publisher.gn1.link/rbm.org.br/pdf/v48n3a12.pdf.

Cassin, J. C. D. (2018). Uso indiscriminado da sibutramina como anorexígeno [TCC]. Cuiabá: Universidade de Cuiabá. https://repositorio.pgsskroton.com/bitstream/123456789/20157/1/JESSICA\%20CRISTINA\%20DAMBROS\%20CASSIN.pdf.

Costa, R. H. F., Lima, D. C. S., Rêgo, I. D. A., Pinho, F. C. S., Neiva, L. D. B., Santana, L. S. O. S., Silva, L. C., Costa, M. S., Pereira Júnior, J. L. 2018. Perfil das prescrições de sibutramina em uma rede de drogaria. Revista Interdisciplinar de Ciências Médicas. https://gpicursos.com/interagin/gestor/uploads/trabalhosfeirahospitalarpiaui/37fec210fb61092acd06595a560e9c29.pdf.

Da Anvisa, B. E., \& Brasília, D. F. ANVISA-Agência Nacional de Vigilância Sanitária. https://www.gov.br/anvisa/pt-br/assuntos/noticiasanvisa/2016/anvisa-libera-comercializacao-de-sibutramina-da-ache.

de Farmácia, C. F., Do Estado, C. R. D. F., Acre, D., Amapá, D., \& Amazonas, D. (2004). Conselho Federal de Farmácia. http://www.cff.org.br/noticia.php?id=4517\&titulo=Sibutramina+e+rem\%C3\%A9dios+para+emagrecer\%3A+entenda.

da Silva, A. C., Magalini, A. P., dos Santos, D. C. F., Malfará, W. R., \& Crisci, A. R. (2018). Efeitos da sibutramina sobre o sistema reprodutor feminino de ratas Wistar. Revista Brasileira Multidisciplinar, 21(1), 95-102. https://revistarebram.com/index.php/revistauniara/article/view/579. 
de Souza, S. S. S., Pinheiro, M. T. R. S., Almeida, P. H. R. F., Lemos, L. B., \& da Silveira Lemos, G. (2017). Sibutramina: falhas e incompletude de documentos na prescrição e dispensação. Revista de Atenção à Saúde (ISSN 2359-4330), 15(51), 23-33. https://seer.uscs.edu.br/index.php/revista_ciencias_saude/article/view/4233.

Dutra, J. R., da Fonseca Souza, S. M., \& Peixoto, M. C. (2015). A influência dos padrões de beleza veiculados pela mídia, como fator decisório na automedicação com moderadores de apetite por mulheres no município de Miracema-RJ. Revista Transformar, (7), 194-213. http://www.fsj.edu.br/transformar/index.php/transformar/article/view/40/37.

Gonzaga, J. B., da Silva Siqueira, F., Zamprone, J. T., Franco, B., Constantino, S. K. C., Silva, E. A. J., \& de Oliveira, R. G. (2014). Análise das prescrições de sibutramina dispensadas em drogarias no micípio de Cuiabá-MT, http://revistas.cff.org.br/?journal=infarma\&page=article\&op=view\&path\%5B $\% 5 \mathrm{D}=714 \&$ path $\% 5 \mathrm{~B} \% 5 \mathrm{D}=\mathrm{pdf} \_29$.

Lima, M. V. D. (2017). Estratégias para tratamento farmacológico da obesidade no Brasil: Revisão sistemática de literatura para análise econômica sob perspectiva privada (Doctoral dissertation, Universidade de São Paulo). https://repositorio.usp.br/item/002870175.

Marini, D. C., de Oliveira Silva, L., \& Oliveira, D. C. (2016). Perfil da dispensação e do uso de sibutramina para tratamento da obesidade. Foco: Caderno de estudos e pesquisas, (7). http://revistafoco.inf.br/ index.php/Foco Fimi/ article/view/57/56.

Moreira, F., \& Alves, A. A. (2015). Utilização de anfetaminas como anorexígenos relacionas à obesidade. Revista Científica da FHO UNIARARAS, 3(1). http://www.uniararas.br/revistacientifica/_documentos/art.9-029-2015.pdf.

Oliveira, K. R., Vilela, P. A., Freitas, J. G. A. D., \& dos Santos, U. G. (2016). Sibutramina: efeitos e riscos do uso indiscriminado em obesos. Revista de trabalhos acadêmicos-universo-Goiânia . http://revista.universo.edu.br/index.php?journal=3GOIANIA4\&page=article\&op=view\&path\%5B\%5D=3112.

Radaelli, M., Pedroso, R. C., \& Medeiros, L. F. (2016). Farmacoterapia da obesidade: Benefícios e Riscos. Saúde e Desenvolvimento Humano, 4(1), 101-115. https://revistas.unilasalle.edu.br/index.php/saude_desenvolvimento/article/view/2317-8582.16.23/pdf.

Silva, V. P. D. (2011). O uso de sibutramina no tratamento de pacientes obesos. http://conitec.gov.br/images/Consultas/Dossie/2019/Dossie_Abeso_25000_091039_2019_77.pdf.

Santos, C. D. S. C., \& Belo, R. F. C. (2017). Prevalência do uso de Fármacos para o Emagrecimento em Universitárias de Sete Lagoas-MG. Revista Brasileira de Ciências da Vida, 5(1). file:///C:/Users/LENOVO/Downloads/523-Texto\%20do\%20artigo-1190-1-10-20170809.pdf.

Silva, L.S, Silva, T.R. Moreira, D.M, Silva, D.R, Martins, S.H.R. (2018). Automedicação com finalidade estética: um estudo de prevalência entre estudantes universitários. Revista LeiaCby, 1(1), 56-67. http://docplayer.com.br/139883601-Automedicacao-com-finalidade-estetica-um-estudo-de.html.

Silva, V. P. D. (2011). O uso de sibutramina no tratamento de pacientes obesos. http://repositorio.faema.edu.br.

Silva, W. D. D. (2018). Efeitos colaterais do uso de Sibutramina contra a obesidade. http://repositorio.fucamp.com.br/handle/FUCAMP/334. 